derived GFR of $55(20-115) \mathrm{mL} / \mathrm{min} / 1.73 \mathrm{~m}^{2}, \mathrm{p}<0.0001$. Median PCR was 55 (36-189) compared to 17 (13-29) in the AKI and NonAKI groups, $p<0.0005$. Liver disease severity scores were not significantly different between AKI groups for both CTP ( $p=0.86)$ or model for end-stage liver disease (MELD, $p=0.14$ ). Plasma cystatin $C$ at $48 \mathrm{~h}$ prior to AKI predicted its subsequent development with an AUROC of 0.82 (sensitivity $86 \%$, specificity $75 \%, p=0.004$, cut off $1.18 \mathrm{mg} / \mathrm{l}$ ). A random urine PCR of $>30$ predicted AKI during that hospital admission with an AUROC of 0.72 (sensitivity, $77 \%$, specificity $63 \%, \mathrm{p}=0.001$ ). Plasma cystatin $\mathrm{C}$ at $48 \mathrm{~h}$ prior to AKI predicted death with an AUROC of 0.88 (sensitivity $100 \%$, specificity $76 \%, \mathrm{p} \leq 0.0001$, cut off $1.18 \mathrm{mg} / \mathrm{l})$. PCR $>49$ predicted the need for RRT with an AUROC of 0.85 (sensitivity $73 \%$, specificity $86 \%$, $\mathrm{p} \leq 0.0001$ ).

Conclusion Estimated GFR using serum creatinine based equations overestimates GFR, therefore an accurate assessment requires a gold standard measure, like iohexol, in patients with advanced liver disease. Cystatin C and the widely available urine PCR measurement can be used to assess the risk of AKI. They both demonstrate potential for predicting AKI $48 \mathrm{~h}$ prior to onset, the need for RRT and hospital survival.

Competing interests None declared.

REFERENCE

1. Slack A, Yeoman A, Wendon J. Renal dysfunction in chronic liver disease. Crit Care 2010;14:214.

\section{PTU-019 METHADONE USE IS ASSOCIATED WITH THE DEVELOPMENT OF COMMON BILE DUCT DILATATION AMONG PATIENTS WITH HEPATITIS C: RESULTS OF A RETROSPECTIVE COHORT STUDY}

doi:10.1136/gutjnl-2012-302514c.19

A Ahmed, ${ }^{*}$ A Stanley, E Forrest, R Gillespie, M Neilson, S Barclay. Glasgow Royal Infirmary, Glasgow, UK

Introduction The synthetic opioid Methadone is widely used in the treatment of opioid dependence. Opioids are known to induce spasm of the sphincter of Oddi, increase common bile duct (CBD) pressures and induce bile duct dilatation. A prior study has shown methadone use to be associated with asymptomatic CBD dilatation among patients with viral hepatitis. We aimed to examine the prevalence of $\mathrm{CBD}$ dilatation among methadone users with hepatitis $\mathrm{C}(\mathrm{HCV})$ at first attendance and subsequent liver clinic follow-up, in comparison with a control group.

Methods Patients with chronic HCV attending our institution between 2003 and 2010 were identified from the Scottish HCV Database. Age, gender, methadone use, and CBD dilatation $(\geq 8 \mathrm{~mm}$ ) identified on initial and follow-up abdominal ultrasound scan (AUS) were recorded. Statistical analysis was performed using SPSS to compare methadone users, vs a control group not on methadone.

Results 618 patients were identified, 316 (51.1\%) on methadone and $302(48.9 \%)$ not. Mean age (42.3 vs $48.5, \mathrm{p}=0.99)$ and gender $(71.5 \%$ male vs $69.2 \%, p=0.53$ ) were similar in the methadone group vs controls. CBD dilatation on initial AUS was significantly higher among the methadone group (47/316 (14.9\%) vs 18/302 (6\%), $\mathrm{p}=0.0003)$. Post cholecystectomy CBD dilatation was uncommon $(4 / 47(8.5 \%)$ vs $3 / 18(16.6 \%), p=0.38)$. Of those with a normal initial CBD, $111 / 269$ (41.3\%) methadone patients and 115/284 $(40.5 \%)$ controls had an interval scan. Over similar durations of follow-up (38.1 months vs 45 months controls, $p=0.53$ ), methadone use was associated with increased de-novo CBD dilatation (15/111 $(13.5 \%)$ vs $6 / 115$ (5.2\%), p=0.03). Rates of subsequent biliary investigation (MRCP/ERCP) were low (12/62 (19.3\%) and $7 / 24$
(29.2\%). An obstructive cause of biliary dilatation was infrequently found among methadone receiving patients and controls (1/12 (8.3\%) vs $1 / 7(14.3 \%), p=0.49)$. No obstructive biliary pathology was found among patients with normal alkaline phosphatase (ALP) in either group.

Conclusion Our study confirms the association between methadone use and $\mathrm{CBD}$ dilatation among patients referred for assessment of HCV. For the first time we have demonstrated an increased rate of new CBD dilatation among methadone users on longitudinal follow-up. Given that one in five patients on methadone demonstrated CBD dilatation during initial assessment or follow-up, with no alternate cause identified among those with a normal ALP, further investigation of these patients may not be necessary. Further work is required to establish and validate algorithms identifying those patients receiving methadone with CBD dilatation in whom further investigation can be safely omitted.

Competing interests None declared.

\section{PTU-020 RIFAXAMIN IS A HIGHLY EFFICACIOUS TREATMENT FOR THE PARKINSONIAN PHENOTYPE OF HEPATIC ENCEPHALOPATHY (HE)}

doi:10.1136/gutjnl-2012-302514c.20

${ }^{1} \mathrm{~B}$ Kok, ${ }^{*} \mathrm{M}$ McPhail, ${ }^{1,2} \mathrm{M}$ Foxton, ${ }^{1} \mathrm{D}$ Shawcross. ${ }^{1}$ Institute of Liver Studies, King's College Hospital, London, UK; ${ }^{2}$ Department of Gastroenterology, Chelsea \& Westminster Hospital, London, UK

Introduction Patients who develop Parkinsonian symptoms on a background of cirrhosis and portosystemic shunting (PSS) form a unique subset of so-called acquired hepatocerebral degeneration. The syndrome is entirely different from acute $\mathrm{HE}$ and other forms of Parkinsonism that develop in patients without liver disease and rarely responds to standard treatments for HE. Rifaxamin is a nonabsorbable antibiotic which has recently been shown to be efficacious in the secondary prevention of recurrent HE and is postulated to decrease gut ammonia production and/or bacterial translocation. Methods To prospectively evaluate the efficacy of rifaxamin $600 \mathrm{mg}$ twice daily in three patients referred to the HE clinic at our institution with advanced cirrhosis, evidence of PSS and debilitating HE with extrapyramidal symptoms including resting tremor, bradykinesia, cog-wheel rigidity, drooling, loss of facial expression, shuffling gait and excessive somnolence. Each patient was evaluated independently by a hepatologist and a neurologist. Neuropsychological function testing (Trails $\mathrm{A}$ and $\mathrm{B}$ test), random venous ammonia $\left(\mathrm{NH}_{3}\right)$, EEG and MRI brain/DaTscan were performed pre- and 4 weeks post rifaxamin.

Results Patient 1 [male, age 61, $\alpha 1 \mathrm{AT}, \mathrm{NH}_{3} 76 \mu \mathrm{mol} / \mathrm{l}$ ] was unable to complete Trails $\mathrm{A} / \mathrm{B}$ test at baseline. On rifaxamin his severe bradykinetic rigidity syndrome, drooling and leaning to one side on walking resolved. His repeat Trails B test was in $75-90^{\text {th }}$ centile for a normal healthy age-matched population. His symptoms improved further on long acting dopamine therapy. Patient 2 [female, age 64, alcohol, abstinent, $\left.\mathrm{NH}_{3} 67 \mu \mathrm{mol} / \mathrm{l}\right]$ had an improved Trails A from the $10^{\text {th }}$ to $50^{\text {th }}$ centile, with resolution of bradykinesia, resting tremor and a dramatic reduction in her somnolence. Patient 3 [male, age 66, alcohol, abstinent, $\left.\mathrm{NH}_{3} 67 \mu \mathrm{mol} / \mathrm{l}\right]$ had remarkable improvement in his asymmetric bradykinetic rigid syndrome, regained his facial expression and was mobile with assistance whereas previously he had required hoisting. None of the patients had any improvement in their ammonia level or EEG with rifaximin despite resolution of symptoms. In the first two patients MRI brain post rifaxamin showed no change (high T1 signal in the globus pallidus) and in patient 3 a DaTscan post rifaxamin still showed decreased uptake in the right corpus striatum, in spite of dramatic clinical improvements. Patient 1 has now been transplanted and his 